PROSTATE CANCER

\section{Epigenetic AR regulation}

A new study in Cancer Cell describes an epigenetic mechanism that increases androgen receptor $(A R)$ transcription in castration-resistant prostate cancer (CRPC) via the nonreceptor tyrosine kinase ACK-1, WD repeat-containing protein 5 (WDR5), and histone-lysine $\mathrm{N}$-methyltransferase 2D (KMT2D, also known as MLL2). An ACK-1-specific inhibitor suppressed both $A R$ and $A R-V 7$ levels and reduced in vivo CRPC tumour growth.

ACK-1 expression had previously been shown to correlate with prostate cancer progression to castration resistance and poor prognosis. Mahajan et al. investigated epigenetic mechanisms in CRPC pathogenesis and found phosphorylation of tyrosine 88 in histone $\mathrm{H} 4(\mathrm{pY} 88-\mathrm{H} 4)$ in three of five tested CRPC samples and predominantly in ACK-1-positive cells. Suppression of ACK-1 through knockdown or the inhibitor $(R)$-9bMS abrogated pY88-H4. pY88-H4 marks were found in distinct regions upstream of $A R$ that seemed to act as enhancers of $A R$.

Further studies in prostate cancer cell lines showed that ACK-1 activity was required for $A R$ expression in an androgen-deprived setting. In addition, ACK-1 inhibition suppressed both AR and $A R-V 7$ expression and had effects on DNA repair, transcription factor E2F, and MYC target gene signatures.

Evaluation of the epigenetic mechanism revealed that a complex of WDR5-MLL2 recognizes $\mathrm{pY} 88$ - $\mathrm{H} 4$ marks and creates transcriptionally activating $\mathrm{H} 3 \mathrm{~K} 4 \mathrm{me} 3$ marks that promote $A R$ transcription.

Analyses of clinical samples suggested that $\mathrm{pY} 88-\mathrm{H} 4, \mathrm{H} 3 \mathrm{~K} 4 \mathrm{me} 3$, and AR expression increase during prostate cancer progression. In mouse xenograft models of CRPC, $(R)$-9bMS treatment halted tumour growth. The authors suggest that an epigenetic circuit of ACK1-pY88-H4WDR5-MLL2-AR drives CRPC and is required to maintain the malignant state.

Clemens Thoma

ORIGINAL ARTICLE Mahajan, K. et al. ACK1/TNK2 regulates histone $\mathrm{H} 4$ Tyr88-phosphorylation and AR gene expression in castration-resistant prostate cancer. Cancer Cell http://dx.doi. org/10.1016/j.ccell.2017.05.003 (2017)
ACK-1 activity was required for AR expression in an androgendeprived setting

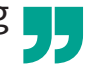

